\title{
Active Sliding Mode Control Antisynchronization of Chaotic Systems with Uncertainties and External Disturbances
}

\author{
Wafaa Jawaada, ${ }^{1}$ M. S. M. Noorani, ${ }^{2}$ and M. Mossa Al-sawalha ${ }^{3}$ \\ ${ }^{1}$ School of Mathematical Sciences, Universiti Kebangsaan Malaysia, Selangor, 43600 Bangi, Malaysia \\ ${ }^{2}$ School of Mathematical Sciences, Universiti Kebangsaan Malaysia,Selangor, 43600 Bangi, Malaysia \\ ${ }^{3}$ Mathematics Department, Faculty of Science, University of Hail, Hail 81451, Saudi Arabia \\ Correspondence should be addressed to Wafaa Jawaada, wafaibrahimj@gmail.com
}

Received 25 August 2011; Revised 22 November 2011; Accepted 30 November 2011

Academic Editor: Debasish Roy

Copyright (C) 2012 Wafaa Jawaada et al. This is an open access article distributed under the Creative Commons Attribution License, which permits unrestricted use, distribution, and reproduction in any medium, provided the original work is properly cited.

\begin{abstract}
The antisynchronization behavior of chaotic systems with parametric uncertainties and external disturbances is explored by using robust active sliding mode control method. The sufficient conditions for achieving robust antisynchronization of two identical chaotic systems with different initial conditions and two different chaotic systems with terms of uncertainties and external disturbances are derived based on the Lyapunov stability theory. Analysis and numerical simulations are shown for validation purposes.
\end{abstract}

\section{Introduction}

Chaotic nonlinear systems appear ubiquitously in nature and can occur inman-made systems. These systems are recognized by their great sensitivity to initial conditions. Many scientists who are interested in this field have struggled to achieve the synchronization or antisynchronization of different chaotic systems, mainly due to its potential applications especially in chemical reactions, power converters, biological systems, information processing, secure communications, and so forth [1-7]. But due to its complexities, such tasks are always difficult to achieve. It is much more attractive and challenging to realize the synchronization or antisynchronization of two different chaotic systems especially if terms of uncertainty are considered. A wide variety of approaches have been proposed for the synchronization or antisynchronization of chaotic systems, which include generalized active control [8-10], nonlinear control [11, 12], adaptive control [13-17], and sliding mode control [18, 19]. Most of the above-mentioned works did not consider the uncertainty of parameters and its effects on the systems. The aim of this paper is to further develop the state observer method for 
constructing antisynchronized slave system for chaotic systems with parametric uncertainties and external disturbances by using a robust active sliding mode method.

The outline of the rest of the paper is organized as follows. Firstly, in Section 2 we present a novel active sliding mode controller design and analysis. Section 3 presents a brief description of the two systems, Next, in Section 4, the active sliding mode control method is applied to antisynchronize two identical chaotic systems with different initial conditions and terms of uncertainties and external disturbances. In Section 5, we apply our method to antisynchronize two different chaotic systems, namely, the chaotic Lü and Genesio systems. Finally, Section 6 provides a summary of our results.

\section{Active Sliding Mode Controller Design and Analysis}

Design procedure of the active sliding mode controller which is a combination of the active controller and the sliding mode controller is given first, and then the stability issue of the proposed method is discussed.

\subsection{Active Sliding Mode Controller Design}

Consider a chaotic system described by the following nonlinear differential equation:

$$
\dot{x}=\left(A_{1}+\Delta A_{1}\right) x+f_{1}(x)+D_{1}(t),
$$

where $x(t) \in R^{n}$ denotes the system's $n$-dimensional state vector, $A_{1} \in R^{n \times n}$ represents the linear part of the system dynamics, and $f_{1}: R^{n} \rightarrow R^{n}$ is the nonlinear part of the system, $\Delta A_{1} \in R^{n \times n}$ is the matrix of uncertainties, and $D_{1}(t) \in R^{n}$ is a vector representing external disturbances. Relation (2.1) represents the master system. The controller $u(t) \in R^{n}$ is added into the slave system, so it is given by

$$
\dot{y}=\left(A_{2}+\Delta A_{2}\right) y+f_{2}(y)+D_{2}(t)+u(t)
$$

where $y(t) \in R^{n}$ is the slave system's $n$-dimensional state vector, $A_{2} \in R^{n \times n}$ and $f_{2}: R^{n} \rightarrow R^{n}$ play similar roles as $A_{1}$ and $f_{1}$ for the master system, $\Delta A_{2} \in R^{n \times n}$ is the matrix uncertainties, and $D_{2}(t) \in R^{n}$ is a vector representing external disturbances. If $A_{1}=A_{2}$ and $f_{1}(\cdot)=f_{2}(\cdot)$, then $x$ and $y$ are the states of two identical chaotic systems. Otherwise they represent the states of two different chaotic systems. The antisynchronization problem is to design the controller $u(t) \in R^{n}$ which antisynchronizes the states of the master and slave systems. The dy-nam-ics of the antisynchronization errors can be expressed as

$$
\begin{aligned}
\dot{e} & =\left(A_{2}+\Delta A_{2}\right) y+f_{2}(y)+\left(A_{1}+\Delta A_{1}\right) x+f_{1}(x)+D_{1}(t)+D_{2}(t)+u(t) \\
& =\left(A_{2}+\Delta A_{2}+\Delta A_{1}\right) e+D_{1}(t)+D_{2}(t)+F(x, y)+u(t),
\end{aligned}
$$

where $e=y+x$ and $F(x, y)=f_{2}(y)+f_{1}(x)+\left(A_{1}-\left(A_{2}+\Delta A_{2}\right)\right) x-\Delta A_{1} y$. Our goal is to design the controller $u(t) \in R^{n}$ such that

$$
\lim _{t \rightarrow \infty}\|e\|=\lim _{t \rightarrow \infty}\left\|y\left(t, y_{0}\right)+x\left(t, x_{0}\right)\right\|=0,
$$


where $\|\cdot\|$ is the Euclidean norm. As the trajectories of the chaotic systems are always bounded, then one can assume uncertainties to be bounded, so, in general,

$$
\begin{aligned}
\left|\Delta A_{1}\right|<\rho, & & \left|\Delta A_{2}\right|<\mu, \\
\left|D_{1}\right|<\delta, & & \left|D_{2}\right|<\epsilon,
\end{aligned}
$$

where $\rho, \mu, \delta$, and $\epsilon$ are positive constants. According to the active control design procedure, one uses the control input $u(t)$ to eliminate the nonlinear part of the error dynamics. In other words, the input vector is considered as

$$
u(t)=H(t)-F(x, y)
$$

The error system (2.3) is then rewritten as

$$
\dot{e}=\left(A_{2}+\Delta A_{2}+\Delta A_{1}\right) e+D_{1}(t)+D_{2}(t)+H(t) .
$$

Equation (2.7) describes the error dynamics with a newly defined control input $H(t)$. There are many possible choices for the control $H(t)$. Without loss of generality, we choose the sliding mode control law as follows:

$$
H(t)=K w(t),
$$

where $K=\left[k_{1}, k_{2}, k_{3}\right]^{T}$ is a constant gain vector and $w(t) \in R$ is the control input that satisfies

$$
w(t)= \begin{cases}w^{+}(t), & s(e) \geq 0 \\ w^{-}(t), & s(e)<0\end{cases}
$$

and $s=s(e)$ is a switching surface which prescribes the desired dynamics. The resultant error dynamics is then

$$
\dot{e}=\left(A_{2}+\Delta A_{2}+\Delta A_{1}\right) e+D_{1}(t)+D_{2}(t)+K w(t) .
$$

In what follows, the appropriate sliding mode controller will be designed according to the sliding mode control theory.

\subsubsection{Sliding Surface Design}

The sliding surface can be defined as follows:

$$
s(e)=C e,
$$

where $C=\left[c_{1}, c_{2}, c_{3}\right]$ is a constant vector. The equivalent control approach is found by the fact that $\dot{s}(e)=0$ is a necessary condition for the state trajectory to stay on the switching 
surface $s(e)=0$. Hence, when in sliding mode, the controlled system satisfies the following conditions:

$$
\begin{aligned}
& s(e)=0, \\
& \dot{s}(e)=0 .
\end{aligned}
$$

Now, using (2.10), (2.11), and (2.12), one can obtain

$$
\dot{s}(e)=\frac{\partial s(e)}{\partial e} \dot{e}=C\left[\left(A_{2}+\Delta A_{2}+\Delta A_{1}\right) e+D_{1}(t)+D_{2}(t)+K w(t)\right]=0
$$

Solving (2.14) for $w(t)$ results in the equivalent control $w_{\text {eq }}(t)$

$$
w_{\mathrm{eq}}(t)=-(C K)^{-1} C\left[\left(A_{2}+\Delta A_{2}+\Delta A_{1}\right) e(t)+D_{1}(t)+D_{2}(t)\right]
$$

where the existence of $(C K)^{-1}$ is a necessary condition. Replacing for $w(t)$ in (2.10) from $w_{\text {eq }}(t)$ of $(2.15)$, the state equation in the sliding mode is determined as follows:

$$
\dot{e}=\left[I-K(C K)^{-1} C\right]\left[\left(A_{2}+\Delta A_{2}+\Delta A_{1}\right) e+D_{1}(t)+D_{2}(t)\right] .
$$

As long as the system (2.10) has all eigenvalues with negative real parts, it is asymptotically stable.

\subsubsection{Design of the Sliding Mode Controller}

We assume that the constant plus proportional rate reaching law is applied. The reaching law can be chosen such that

$$
\dot{s}=-\frac{q s}{|s|+\gamma}-r s
$$

where $r$ is a positive real number. The gains $q>0$ and $r>0$ are determined such that the sliding condition is satisfied and the sliding mode motion occurred. From (2.10) and (2.11), it can be found that

$$
\dot{s}=C\left[\left(A_{2}+\Delta A_{2}+\Delta A_{1}\right) e+D_{1}(\mathrm{t})+D_{2}(t)+K w(t)\right] .
$$

Now, from (2.17) and (2.18), the control input is determined as

$$
w(t)=-(C K)^{-1}\left[C\left(r I+\left(A_{2}+\Delta A_{2}+\Delta A_{1}\right)\right) e(t)+C D_{1}(t)+C D_{2}(t)+\frac{q s}{|s|+\gamma}\right] .
$$




\subsubsection{Stability Analysis}

To check the stability of the controlled system, one can consider the following Lyapunov candidate function:

$$
V=\frac{1}{2} s^{2}
$$

The time derivative of $(2.21)$ is

$$
\dot{V}=\dot{s} s=-\frac{q s^{2}}{|s|+\gamma}-r s^{2}
$$

Since $s^{2} /(|s|+\gamma)>0, r>0$ and $q>0$, we have $\dot{V}=\dot{s} s<0$; therefore, $\dot{V}(e)$ is negative definite. This property implies boundedness of the sliding surface $s$. The error dynamics can be obtained using (2.19) in (2.10):

$$
\begin{aligned}
\dot{e}= & {\left[\left(A_{2}+\Delta A_{2}+\Delta A_{1}\right)-K(C K)^{-1} C\left(r I+\left(A_{2}+\Delta A_{2}+\Delta A_{1}\right)\right)\right] e } \\
& -K(C K)^{-1} C\left[D_{1}(t)+D_{2}(t)\right]-K(K C)^{-1} \frac{q s}{|s|+\gamma} .
\end{aligned}
$$

As a linear system with bounded input $\left(-K(C K)^{-1} q\right.$ for $s \geq 0$ and $K(C K)^{-1} q$ for $\left.s<0\right)$, the error system is asymptotically stable if and only if $\left[\left(A_{2}+\Delta A_{2}+\Delta A_{1}\right)-K(C K)^{-1} C\left(r I+\left(A_{2}+\right.\right.\right.$ $\left.\left.\left.\Delta A_{2}+\Delta A_{1}\right)\right)\right]$ has negative eigenvalues. Because of the special structure for matrix $A_{2}$ in the given chaotic systems, one of the eigenvalues is always $-r$ and therefore is stable. The two other eigenvalues are independent from $r$ and determined by the other control parameters that is, $K$ and $C$. The latter two eigenvalues can be negative or positive depending on $K$ and $C$ values. By appropriate choices of $r, K$, and $C$, one is able not only to stabilize the error system but also to adjust the rate of the error convergence. The parameter $q$ can be used to enhance the robust property expected from a sliding mode controller.

\section{Systems Description}

The Lü attractor, which connects the Lorenz attractor [20] andChen and Ueta attractor [21, 22] and represents the transition from one to another, was proposed and analyzed by lü et al. [23]. The Lü chaotic system is described by the following system of differential equations:

$$
\begin{aligned}
& \dot{x}=a(y-x), \\
& \dot{y}=-x z+c y, \\
& \dot{z}=x y-b z,
\end{aligned}
$$


where $x, y$, and $z$ are state variables and $a, b$, and $c$ are positive parameters. This system is dissipative for $c<a+b$ and has a chaotic attractor when $a=36, b=3$, and $c=20$. The Genesio system was introduced by Genesio and Tesi in [24]. It is given by

$$
\begin{aligned}
& \dot{x}=y, \\
& \dot{y}=z, \\
& \dot{z}=-c x-b y-a z+x^{2},
\end{aligned}
$$

where $x, y$, and $z$ are state variables, and $a, b$, and $c$ are the positive real constants satisfying $a b<c$. Throughout this paper, we set $a=1.2, b=2.92$, and $c=6$ such that the system exhibits chaotic behavior.

\section{Active Sliding Mode Antisynchronization between Two Identical Systems}

In order to observe synchronization behavior between two identical chaotic systems via active sliding mode control, we consider two examples, the first one is Lü system in (3.1) and the second example is Genesio system which is described in (3.2).

\subsection{Active Sliding Mode Antisynchronization between Two Identical Lü Systems}

For the Lü system, let us consider that

$$
\Delta A=\left(\begin{array}{ccc}
0 & 1 & 0 \\
-0.8 & 0 & -1 \\
0 & 0 & -3
\end{array}\right), \quad D(t)=\left(\begin{array}{c}
0.1 \cos (50 t) \\
-0.1 \cos (50 t) \\
0.1 \sin (50 t)
\end{array}\right)
$$

The master system and the slave system can be written as the following respectively:

$$
\begin{aligned}
& \dot{x}_{1}=a\left(y_{1}-x_{1}\right)+y_{1}+0.1 \cos (50 t) \\
& \dot{y}_{1}=-x_{1} z_{1}+c y_{1}-0.8 x_{1}-z_{1}-0.1 \cos (50 t) \\
& \dot{z}_{1}=x_{1} y_{1}-b z_{1}-3 z_{1}+0.1 \sin (50 t)
\end{aligned}
$$

and the slave system can be written as

$$
\begin{aligned}
& \dot{x}_{2}=a\left(y_{2}-x_{2}\right)+y_{2}+0.1 \cos (50 t)+u_{1} \\
& \dot{y}_{2}=-x_{2} z_{2}+c y_{2}-0.8 x_{2}-z_{2}-0.1 \cos (50 t)+u_{2} \\
& \dot{z}_{2}=x_{2} y_{2}-b z_{2}-3 z_{2}+0.1 \sin (50 t)+u_{3}
\end{aligned}
$$


where $u_{1}, u_{2}, u_{3}$ are three control functions to be designed in order to determine the control functions and to realize the active sliding mode antisynchronization between the systems in (4.2) and (4.3). We add (4.2) and (4.3) to get

$$
\begin{aligned}
& \dot{e}_{1}=a\left(e_{2}-e_{1}\right)+e_{2}+0.2 \cos (50 t)+u_{1}, \\
& \dot{e}_{2}=c e_{2}-0.8 e_{1}-e_{3}-x_{1} z_{1}-x_{2} z_{2}-0.2 \cos (50 t)+u_{2}, \\
& \dot{e}_{3}=-(b+3) e_{3}+x_{2} y_{2}+x_{1} y_{1}+0.2 \sin (50 t)+u_{3},
\end{aligned}
$$

where $e_{1}=x_{2}+x_{1}, e_{2}=y_{2}+y_{1}$, and $e_{3}=z_{2}+z_{1}$. Our goal is to find proper control functions $u_{i}(i=1,2,3)$ such that system equation (4.3) globally antisynchronizes system equation (4.2) asymptotically; that is,

$$
\lim _{t \rightarrow \infty}\|e\|=0
$$

where $e=\left[e_{1}, e_{2}, e_{3}\right]^{T}$. Without the controls $\left(u_{i}=0, i=1,2,3\right)$, the trajectories of the two systems equations (4.2) and (4.3), will quickly separate with each other and become irrelevant. However, when controls are applied, the two systems will approach synchronization for any initial conditions by appropriate control functions. if the control parameters are chosen as $C=(0,1,1), K=(1,1,0)^{T}$, and $r=1, q=30$, and $\gamma=0.01$, then the switching surface

$$
\begin{gathered}
s(e)=e_{2}+e_{3} \\
w(t)=-\left[(a+1) e_{2}+(-b-4) e_{3}-0.2 \cos (50 t)+0.2 \sin (50 t)+s+\frac{30 s}{|s|+0.01}\right]
\end{gathered}
$$

so the controllers are:

$$
\begin{aligned}
u_{1}= & -e_{1}-\left[(a+1) e_{2}+(-b-4) e_{3}-0.2 \cos (50 t)+0.2 \sin (50 t)+s+\frac{30 s}{|s|+0.01}\right], \\
u_{2}= & x_{1} z_{1}+x_{2} z_{2}+0.8 e_{1}+e_{3} \\
& -\frac{1}{2}\left[(a+1) e_{2}+(-b-4) e_{3}-0.2 \cos (50 t)+0.2 \sin (50 t)+s+\frac{30 s}{|s|+0.01}\right], \\
u_{3}= & -x_{1} y_{1}-x_{2} y_{2}+3 e_{3} .
\end{aligned}
$$

Applying controller (4.7) to the slave system then the slave system in (4.3) can synchronize master system equation (4.2) asymptotically. To verify and demonstrate the effectiveness of the proposed method, we discuss the simulation result for the active sliding mode antisynchronization between two identical Lü systems. For these simulations, the fourth-order Runge-Kutta method is used to solve the systems with time step size 0.001 . We assumed that the initial conditions, $\left(x_{1}(0), y_{1}(0), z_{1}(0)\right)=(2,2,20)$ and $\left(x_{2}(0), y_{2}(0), z_{2}(0)\right)=(-3,-1,-1)$. Hence the error system has the initial values $e_{1}(0)=-1, e_{2}(0)=1$, and $e_{3}(0)=19$. The systems parameters are chosen as $a=36, b=3, c=20$ in the simulations such that Lü system exhibit chaotic behavior. Antisynchronization of the systems equations (4.3) and (4.2) via active sliding mode controllers in (4.7) is shown in Figure 1. Figures $1(\mathrm{a})-1$ (c) display the state trajectories of master system (4.2) and slave system (4.3). Figure 1(d) displays the error signals $e_{1}, e_{2}, e_{3}$ of the Lü system under the controller equations (4.7). 

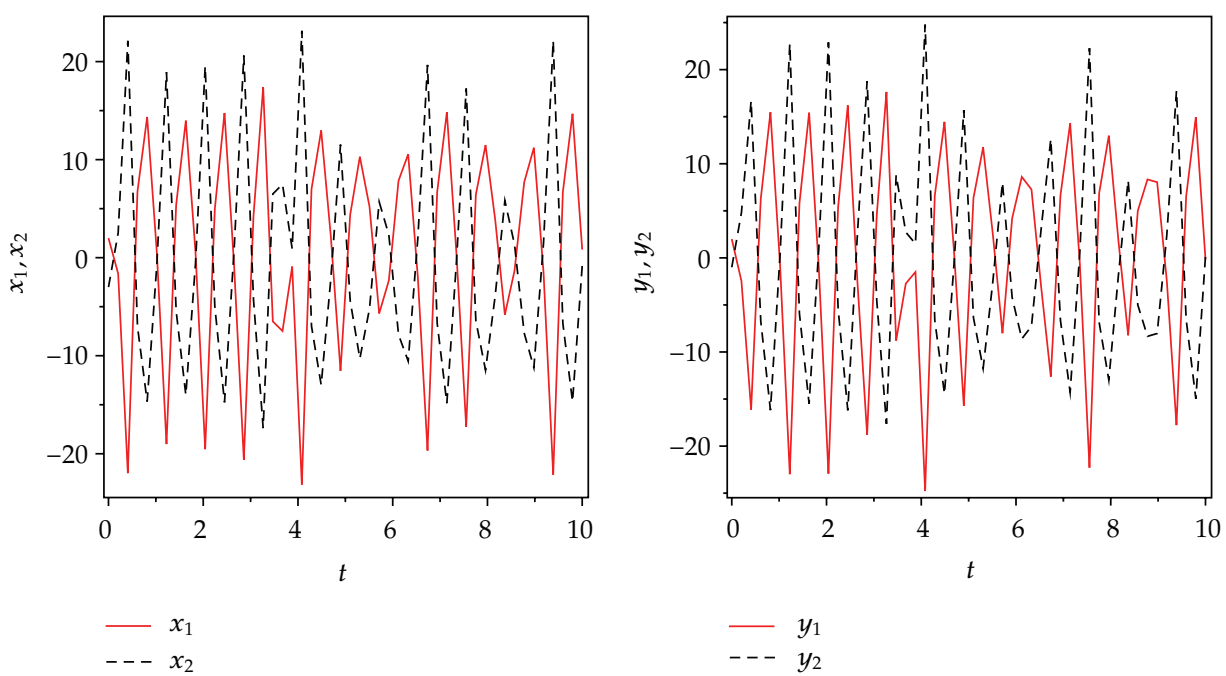

(a)

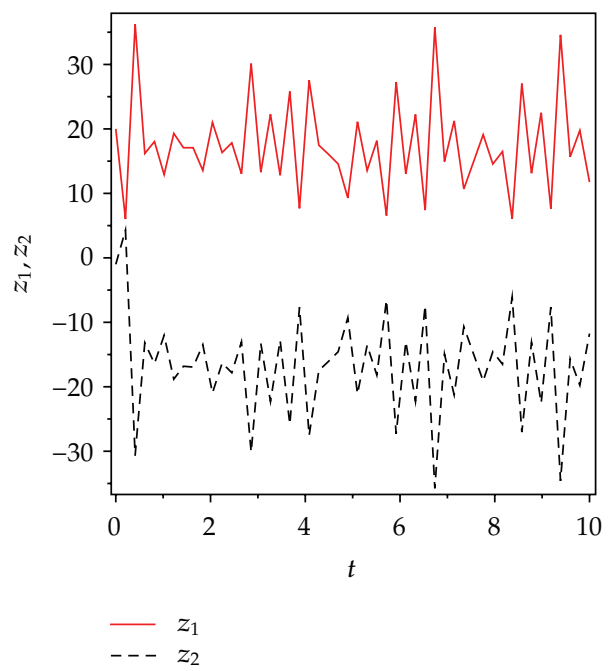

(c)

(d)

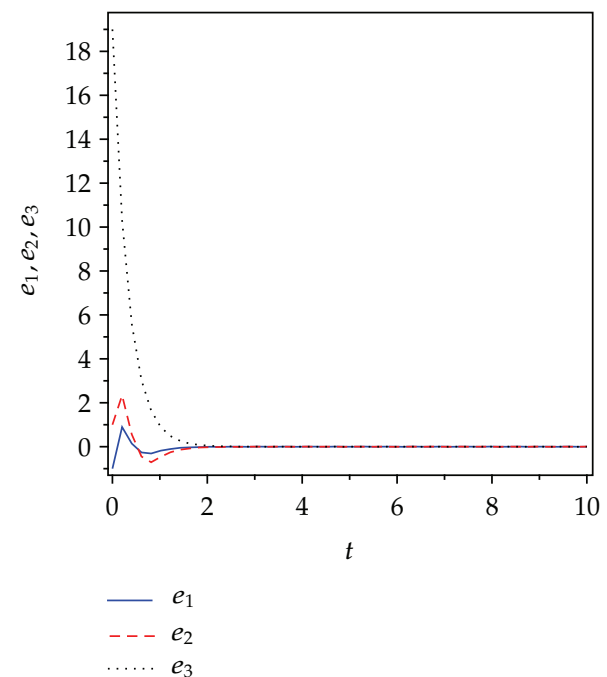

Figure 1: State trajectories of drive system (4.2) and response system (4.3), (a) signals $x_{1}$ and $x_{2} ;$ (b) signals $y_{1}$ and $y_{2} ;$ (c) signals $z_{1}$ and $z_{2} ;$ (d) the error signals $e_{1}, e_{2}, e_{3}$ between two identical Lü systems with different initial conditions under the controller (4.7).

\subsection{Active Sliding Mode Antisynchronization between Two Identical Genesio Systems}

For the Genesio system, let us consider that

$$
\Delta A=\left(\begin{array}{ccc}
0.5 & 0 & 0 \\
0 & -0.3 & 0 \\
-0.5 & 0 & 0
\end{array}\right), \quad D(t)=\left(\begin{array}{c}
0.1 \cos (100 t) \\
0.1 \sin (100 t) \\
0.1 \sin (100 t)
\end{array}\right)
$$


The master system and the the slave system can be written as the following; respectively,

$$
\begin{aligned}
& \dot{x}_{1}=y_{1}+0.5 x_{1}+0.1 \cos (100 t) \\
& \dot{y}_{1}=z_{1}-0.3 y_{1}+0.1 \sin (100 t) \\
& \dot{z}_{1}=-c x_{1}-b y_{1}-a z_{1}+x_{1}^{2}-0.5 x_{1}+0.1 \sin (100 t)
\end{aligned}
$$

and the slave system can be written as

$$
\begin{aligned}
& \dot{x}_{2}=y_{2}+0.5 x_{2}+0.1 \cos (100 t)+u_{1}, \\
& \dot{y}_{2}=z_{2}-0.3 y_{2}+0.1 \sin (100 t)+u_{2} \\
& \dot{z}_{2}=-c x_{2}-b y_{2}-c z_{2}+x_{2}^{2}-0.5 x_{2}+0.1 \sin (100 t)+u_{3}
\end{aligned}
$$

where $u_{1}, u_{2}, u_{3}$ are three control functions to be designed in order to determine the control functions and to realize the active sliding mode antisynchronization between the systems in (4.9) and (4.10). We add (4.9) and (4.10) to get

$$
\begin{aligned}
& \dot{e}_{1}=e_{2}+0.5 e_{1}+0.2 \cos (100 t)+u_{1} \\
& \dot{e}_{2}=e_{3}-0.3 e_{2}+0.2 \sin (100 t)+u_{2} \\
& \dot{e}_{3}=-(c+0.5) e_{1}-b e_{2}-a e_{3}+x_{1}^{2}+x_{2}^{2}+0.2 \sin (100 t)+u_{3}
\end{aligned}
$$

where $e_{1}=x_{2}+x_{1}, e_{2}=y_{2}+y_{1}$ and $e_{3}=z_{2}+z_{1}$. Our goal is to find proper control functions $u_{i}(i=1,2,3)$ such that system equation (4.10) globally antisynchronizes system equation (4.9) asymptotically, that is,

$$
\lim _{t \rightarrow \infty}\|e\|=0,
$$

where $e=\left[e_{1}, e_{2}, e_{3}\right]^{T}$. Without the controls $\left(u_{i}=0, i=1,2,3\right)$, the trajectories of the two systems, (4.9) and (4.10), will quickly separate with each other and become irrelevant. However, when controls are applied, the two systems will approach synchronization for any initial conditions by appropriate control functions. if the control parameters are chosen as $C=(6,0,-1), K=(1,0,0)^{T}$ and $r=1, q=60$, and $\gamma=0.01$, then the switching surface

$$
\begin{gathered}
s(e)=6 e_{1}-e_{3}, \\
w(t)=-\frac{1}{6}\left[-6 c e_{1}-(1-a) e_{3}+1.2 \cos (100 t)-0.2 \sin (100 t)+s+\frac{60 s}{|s|+0.01}\right],
\end{gathered}
$$

so the controllers are

$$
\begin{aligned}
& u_{1}=-0.5 e_{1}-\frac{1}{6}\left[-6 c e_{1}-(1-a) e_{3}+1.2 \cos (100 t)-0.2 \sin (100 t)+s+\frac{60 s}{|s|+0.01}\right], \\
& u_{2}=0.3 e_{2} \\
& u_{3}=+0.5 e_{1}-x_{1}^{2}-x_{2}^{2} .
\end{aligned}
$$


Applying controller (4.14) to the slave system then the slave system, in (4.10) can synchronize master system equation (4.9) asymptotically. In the following, we discuss the simulation results of the proposed method. For these simulations, the fourth-order Runge-Kutta method is used to solve the systems with time step size 0.001 . We assumed that the initial conditions, $\left(x_{1}(0), y_{1}(0), z_{1}(0)\right)=(2,2,2)$ and $\left(x_{2}(0), y_{2}(0), z_{2}(0)\right)=(-2,-3,-3)$. Hence the error system has the initial values $e_{1}(0)=0, e_{2}(0)=-1$ and $e_{3}(0)=-1$. The systems parameters are chosen as $a=1.2, b=2.92$, and $c=6$ in the simulations such that both systems exhibit chaotic behavior. Antisynchronization of the systems equations (4.10) and (4.9) via active sliding mode controllers in (4.14) are shown in Figure 2. Figures 2(a)-2(c) display the state trajectories of master system (4.9) and slave system (4.10). Figure 2(d) display the error signals $e_{1}, e_{2}, e_{3}$ of Genesio system under the controller equations (4.14).

\section{Active Sliding Mode Antisynchronization between Two Different Systems}

In this section the antisynchronization behavior between two different chaotic systems via active sliding mode control is investigated, the Lü system in (3.1) is assumed as the master system and the Genesio system in (3.2) is taken as the slave system. If we take:

$$
\begin{array}{cc}
\Delta A_{1}=\left(\begin{array}{ccc}
0 & 4 & 0 \\
0 & 1 & 0 \\
0.5 & 0 & 0
\end{array}\right), & \Delta A_{2}=\left(\begin{array}{ccc}
-2 & 0 & 5 \\
0 & -1 & 0 \\
1 & 0 & 0
\end{array}\right) \\
D_{1}(t)=\left(\begin{array}{c}
-0.1 \cos (10 t) \\
-0.1 \cos (10 t) \\
-0.2 \sin (10 t)
\end{array}\right), & D_{2}(t)=\left(\begin{array}{c}
0.1 \cos (10 t) \\
-0.1 \cos (10 t) \\
0.2 \sin (10 t)
\end{array}\right) .
\end{array}
$$

Here is the master and slave systems; respectively,

$$
\begin{aligned}
& \dot{x}_{1}=a\left(y_{1}-x_{1}\right)+4 y_{1}-0.1 \cos (10 t), \\
& \dot{y}_{1}=-x_{1} z_{1}+c y_{1}-y_{1}-0.1 \cos (10 t), \\
& \dot{z}_{1}=x_{1} y_{1}-b z_{1}+0.5 x_{1}-0.2 \sin (10 t), \\
& \dot{x}_{2}=y_{2}-2 x_{2}+5 z_{2}+0.1 \cos (10 t)+u_{1}, \\
& \dot{y}_{2}=z_{2}-y_{2}+u_{2}-0.1 \cos (10 t), \\
& \dot{z}_{2}=-c x_{2}-b y_{2}-c z_{2}+x_{2}^{2}+x_{2}+0.2 \sin (10 t)+u_{3} .
\end{aligned}
$$

We add (5.2) to (5.3) to get

$$
\begin{aligned}
& \dot{e}_{1}=e_{2}+a_{1}\left(y_{1}-x_{1}\right)-y_{1}+4 y_{1}-2 x_{2}+5 z_{2}+u_{1} \\
& \dot{e}_{2}=e_{3}+c_{1} y_{1}-x_{1} z_{1}-z_{1}+y_{1}-y_{2}-0.2 \cos (10 t)+u_{2}, \\
& \dot{e}_{3}=-c_{2} e_{1}-b_{2} e_{2}-a_{2} e_{3}+x_{2}^{2}-b_{1} z_{1}+x_{1} y_{1}+c_{2} x_{1}+b_{2} y_{1}+a_{2} z_{1}+0.5 x_{1}+x_{2}+u_{3}
\end{aligned}
$$




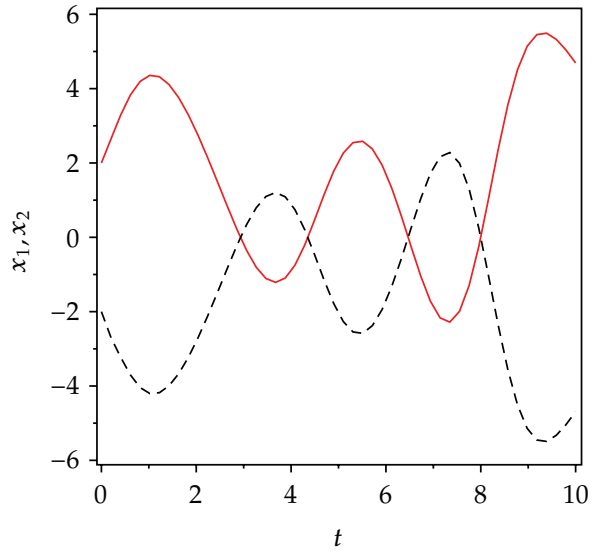

$-x_{1}$

$---x_{2}$

(a)

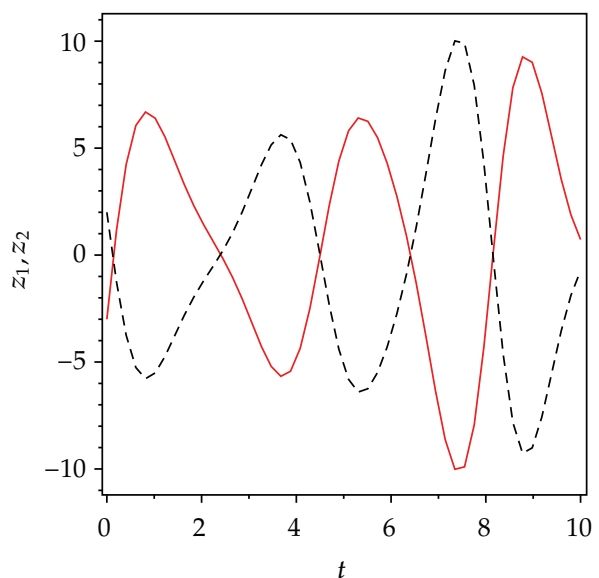

$\begin{array}{ll}- & z_{1} \\ --- & z_{2}\end{array}$

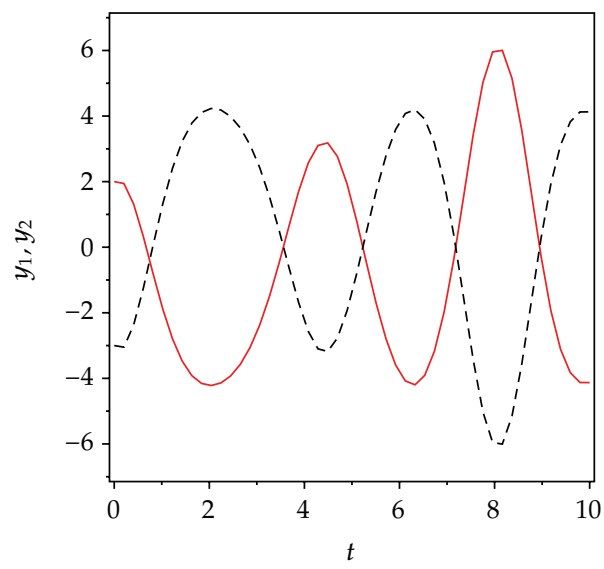

$\begin{array}{ll}- & y_{1} \\ --- & y_{2}\end{array}$

(b)

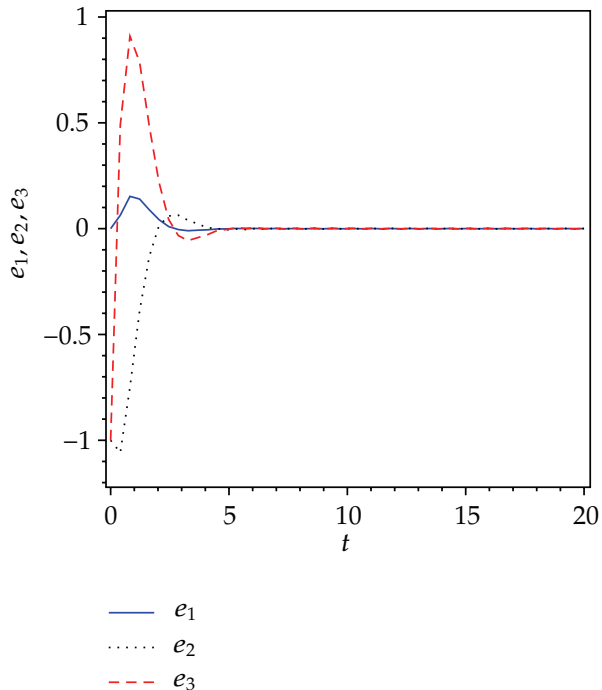

(d)

Figure 2: State trajectories of drive system (4.9) and response system (4.10), (a) signals $x_{1}$ and $x_{2}$; (b) signals $y_{1}$ and $y_{2} ;$ (c) signals $z_{1}$ and $z_{2} ;(\mathrm{d})$ the error signals $e_{1}, e_{2}, e_{3}$ between two identical Genesio systems with different initial conditions under the controller (4.14).

where $e_{1}=x_{2}+x_{1}, e_{2}=y_{2}+y_{1}$, and $e_{3}=z_{2}+z_{1}$. So we are aiming to find proper control functions $u_{i}(i=1,2,3)$ such that system equation (5.3) globally antisynchronizes system equation (5.2) asymptotically; that is,

$$
\lim _{t \rightarrow \infty}\|e\|=0,
$$

where $e=\left[e_{1}, e_{2}, e_{3}\right]^{T}$. 
If the control parameters are chosen as $C=(0,1,-1), K=(0,1,0)^{T}$, and $r=1, q=10$ and $r=0.01$ then the switching surface

$$
\begin{gathered}
s(e)=e_{2}-e_{3} \\
w(t)=\left[\left(5-b_{2}\right) e_{2}-\left(6-a_{2}\right) e_{3}-0.2 \cos (10 t)+s+\frac{10 s}{|s|+0.01}\right],
\end{gathered}
$$

using (5.7) we get

$$
\begin{gathered}
u_{1}=y_{1}-a_{1}\left(y_{1}-x_{1}\right)-2 x_{1}+4 y_{2}+5 z_{1}, \\
u_{2}=x_{1} z_{1}-c_{1} y_{1}+z_{1}-y_{1}+y_{2}-\left[\left(5-b_{2}\right) e_{2}-\left(6-a_{2}\right) e_{3}-0.2 \cos (10 t)+s+\frac{10 s}{|s|+0.01}\right], \\
u_{3}=-x_{1} y_{1}-c_{2} x_{1}-b_{2} y_{1}-b_{2} y_{1}-a_{2} z_{1}+b_{1} z_{1}-x_{2}^{2}+x_{1}+0.5 x_{2} .
\end{gathered}
$$

Applying controllers in (5.8), slave system equation (5.3) can antisynchronize master system equation (5.2) asymptotically. To verify and demonstrate the effectiveness of the proposed method, we discuss the simulation result for the active sliding mode antisynchronization between the Lü system and the Genesio system. In the numerical simulations, the fourthorder Runge-Kutta method is used to solve the systems with time step size 0.001 . For this numerical simulation, we assumed that the initial conditions $\left(x_{1}(0), y_{1}(0), z_{1}(0)\right)=(-3,2,-8)$ and $\left(x_{2}(0), y_{2}(0), z_{2}(0)\right)=(2,3,-2)$. Hence the error system has the initial values $e_{1}(0)=$ $-1, e_{2}(0)=5$, and $e_{3}(0)=-10$. The system parameters are chosen as $a_{1}=36, b_{1}=$ $3, c_{1}=20$, and $a_{2}=1.2, b_{2}=2.92, c_{2}=6$ in the simulations such that both systems exhibit chaotic behavior. Antisynchronization of the systems equations (5.2) and (5.3) via active sliding mode control law in (5.8) are shown in Figure 3. Figures 3(a)-3(c) display the state trajectories of master system (5.2) and slave system (5.3). Figure 3(d) displays the error signals $e_{1}, e_{2}, e_{3}$ of the Lü system and the Genesio system under the controller equations (5.8).

\section{Concluding Remark}

In this paper, we have applied the antisynchronization to some chaotic systems with parametric uncertainties and external disturbances via active sliding mode control. We have proposed a novel robust active sliding mode control scheme for asymptotic chaos antisynchronization by using the Lyapunov stability theory. Finally, the numerical simulations proved the robustness and effectiveness of our method. 


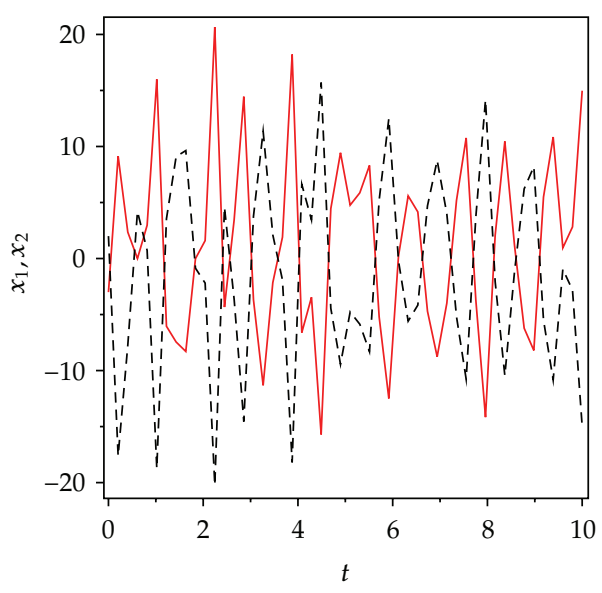

$$
\begin{array}{rr}
- & x_{1} \\
--- & x_{2}
\end{array}
$$

(a)

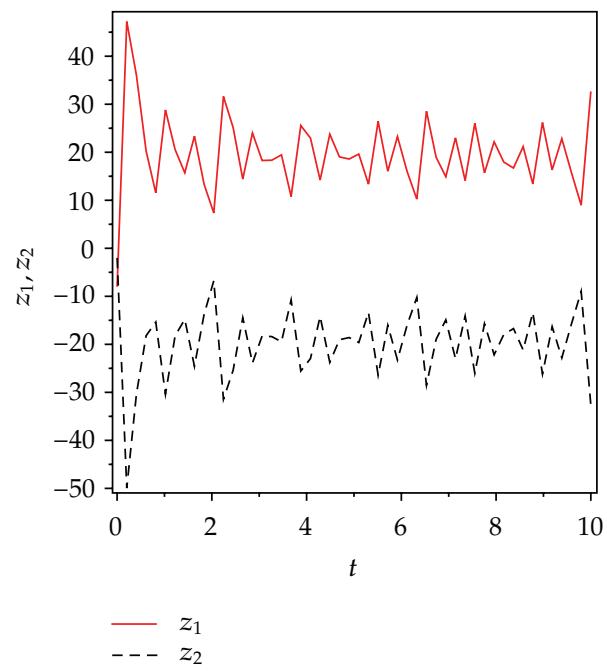

(c)
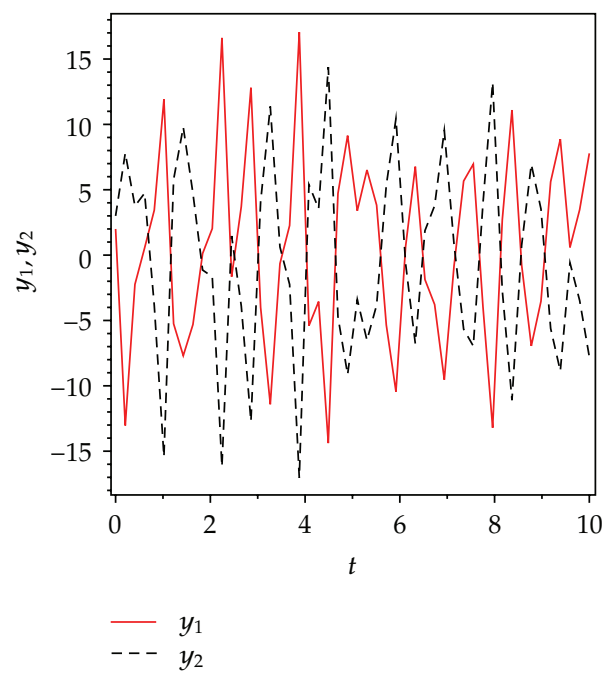

(b)

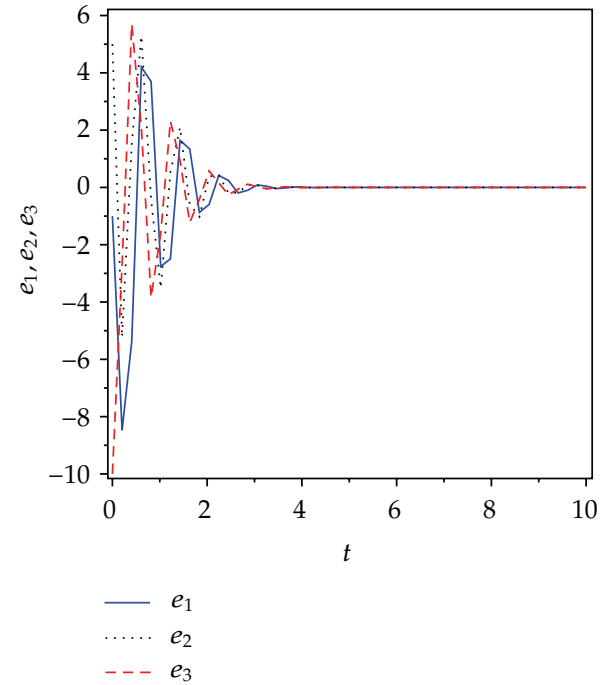

(d)

Figure 3: State trajectories of drive system (5.2) and response system (5.3), (a) signals $x_{1}$ and $x_{2}$; (b) signals $y_{1}$ and $y_{2} ;$ (c) signals $z_{1}$ and $z_{2} ;(\mathrm{d})$ the error signals $e_{1}, e_{2}, e_{3}$ between the Lü system and the Genesio system under the controller (5.8).

\section{Acknowledgment}

This work is financially supported by the Universiti Kebangsaan Malaysia Grant: UKM-DLP2011-016.

\section{References}

[1] L. M. Pecora and T. L. Carroll, "Synchronization in chaotic systems," Physical Review Letters, vol. 64, no. 8, pp. 821-824, 1990. 
[2] G. Chen and X. Dong, From Chaos to Order, vol. 24 of World Scientific Series on Nonlinear Science. Series A: Monographs and Treatises, World Scientific Publishing, Singapore, 1998.

[3] M. Bennett, M. F. Schatz, H. Rockwood, and K. Wiesenfeld, "Huygens's clocks," Proceedings of the Royal Society of London A, vol. 458, no. 2019, pp. 563-579, 2002.

[4] G. Hu, Y. Zhang, H. A. Cerdeira, and S. Chen, "From low-dimensional synchronous chaos to highdimensional desynchronous spatiotemporal chaos in coupled systems," Physical Review Letters, vol. 85, no. 16, pp. 3377-3380, 2000.

[5] M. C. Ho, Y.C. Hung, and C. H. Chou, "Phase and anti-phase synchronization of two chaotic systems by using active control," Physics Letters Section A, vol. 296, no. 1, pp. 43-48, 2002.

[6] A. Uchida, Y. Liu, I. Fischer, P. Davis, and T. Aida, "Chaotic antiphase dynamics and synchronization in multimode semiconductor lasers," Physical Review A, vol. 64, no. 2, pp. 023801-023807, 2001.

[7] G.-H. Li and S.-P. Zhou, "Anti-synchronization in different chaotic systems," Chaos, Solitons and Fractals, vol. 32, no. 2, pp. 516-520, 2007.

[8] M. M. El-Dessoky, "Synchronization and anti-synchronization of a hyperchaotic Chen system," Chaos, Solitons and Fractals, vol. 39, no. 4, pp. 1790-1797, 2009.

[9] M. M. Al-Sawalha and M. S. M. Noorani, "Chaos anti-synchronization between two novel different hyperchaotic systems," Chinese Physics Letters, vol. 25, no. 8, pp. 2743-2746, 2008.

[10] M. M. Al-sawalha and M. S. M. Noorani, "Active anti-synchronization between identical and distinctive hyperchaotic systems," Open Systems and Information Dynamics, vol. 15, no. 4, pp. 371-382, 2008.

[11] M. Mossa Al-sawalha and M. S. M. Noorani, "On anti-synchronization of chaotic systems via nonlinear control," Chaos, Solitons and Fractals, vol. 42, no. 1, pp. 170-179, 2009.

[12] M. M. Al-Sawalha and M. S. M. Noorani, "Anti-synchronization of two hyperchaotic systems via nonlinear control," Communications in Nonlinear Science and Numerical Simulation, vol. 14, no. 8, pp. 3402-3411, 2009.

[13] M. M. Al-sawalha and M. S. M. Noorani, "Anti-synchronization of chaotic systems with uncertain parameters via adaptive control," Physics Letters Section A, vol. 373, no. 32, pp. 2852-2857, 2009.

[14] M. M. Al-sawalha, M. S. M. Noorani, and M. M. Al-dlalah, "Adaptive anti-synchronization of chaotic systems with fully unknown parameters," Computers $\mathcal{E}$ Mathematics with Applications, vol. 59, no. 10, pp. 3234-3244, 2010.

[15] R. Li, W. Xu, and S. Li, "Anti-synchronization on autonomous and non-autonomous chaotic systems via adaptive feedback control," Chaos, Solitons and Fractals, vol. 40, no. 3, pp. 1288-1296, 2009.

[16] J. Horng, H. K. Chen, and Y. K. Lin, "Synchronization and anti-synchronization coexist in Chen-Lee chaotic systems," Chaos, Solitons and Fractals, vol. 39, no. 2, pp. 707-716, 2009.

[17] Q. Song and J. Cao, "Synchronization and anti-synchronization for chaotic systems," Chaos, Solitons and Fractals, vol. 33, no. 3, pp. 929-939, 2007.

[18] M. Zribi, N. Smaoui, and H. Salim, "Synchronization of the unified chaotic systems using a sliding mode controller," Chaos, Solitons and Fractals, vol. 42, no. 5, pp. 3197-3209, 2009.

[19] Y. Zhao and W. Wang, "Chaos synchronization in a Josephson junction system via active sliding mode control," Chaos, Solitons and Fractals, vol. 41, no. 1, pp. 60-66, 2009.

[20] E. N. Lorenz, "Deterministic nonperiodic flow," Journal of the Atmospheric Sciences, vol. 20, no. 2, pp. 130-141, 1963.

[21] G. Chen and T. Ueta, "Yet another chaotic attractor," International Journal of Bifurcation and Chaos in Applied Sciences and Engineering, vol. 9, no. 7, pp. 1465-1466, 1999.

[22] J. C. Sprott, Chaos and Time-Series Analysis, Oxford University Press, Oxford, UK, 2003.

[23] J. Lü, G. Chen, and S. Zhang, "Dynamical analysis of a new chaotic attractor," International Journal of Bifurcation and Chaos in Applied Sciences and Engineering, vol. 12, no. 5, pp. 1001-1015, 2002.

[24] R. Genesio and A. Tesi, "Harmonic balance methods for the analysis of chaotic dynamics in nonlinear systems," Automatica, vol. 28, no. 3, pp. 531-548, 1992. 


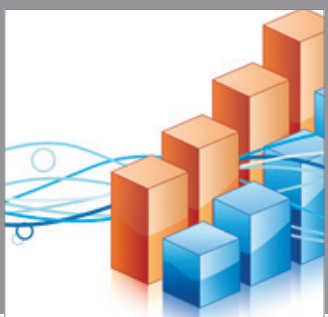

Advances in

Operations Research

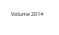

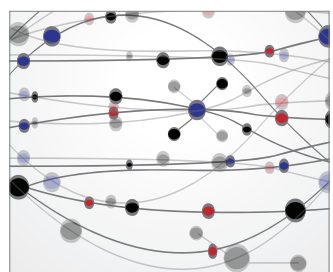

\section{The Scientific} World Journal
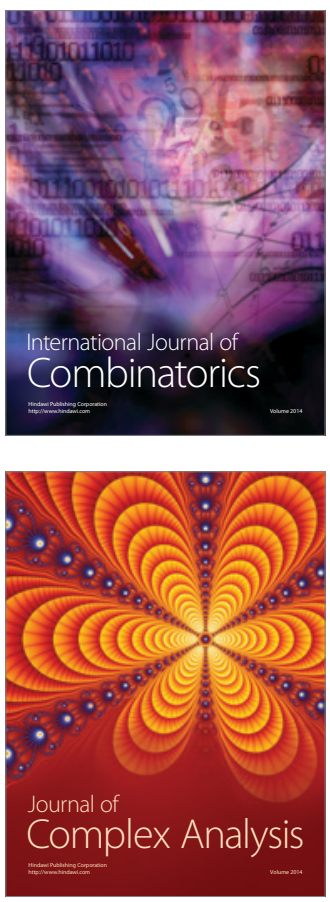

International Journal of

Mathematics and

Mathematical

Sciences
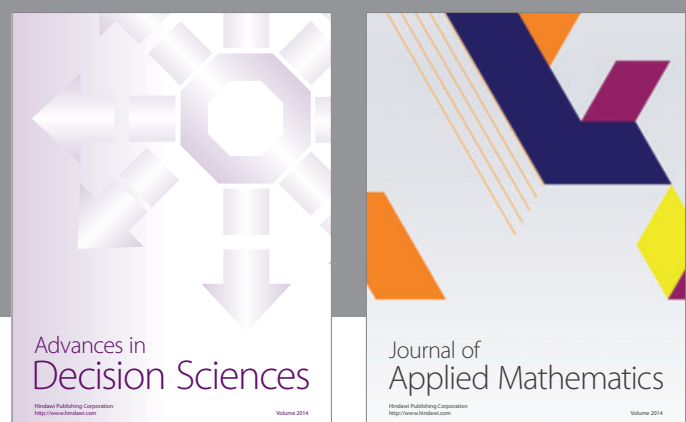

Journal of

Applied Mathematics
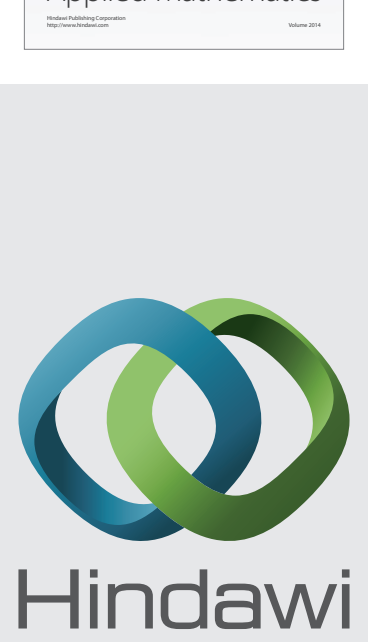

Submit your manuscripts at http://www.hindawi.com
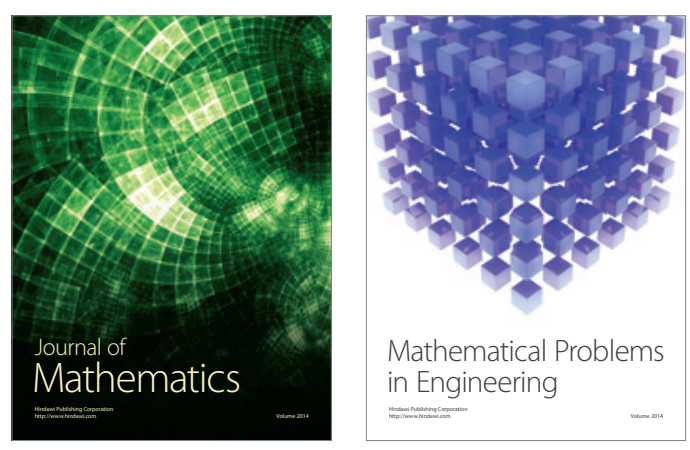

Mathematical Problems in Engineering
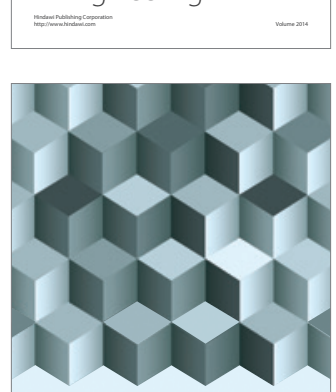

Journal of

Function Spaces
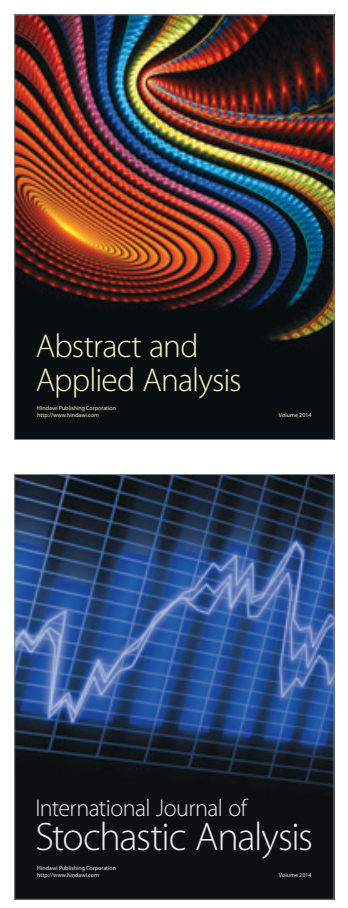

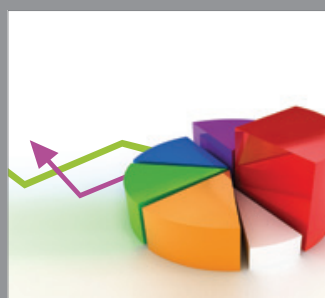

ournal of

Probability and Statistics

Promensencen
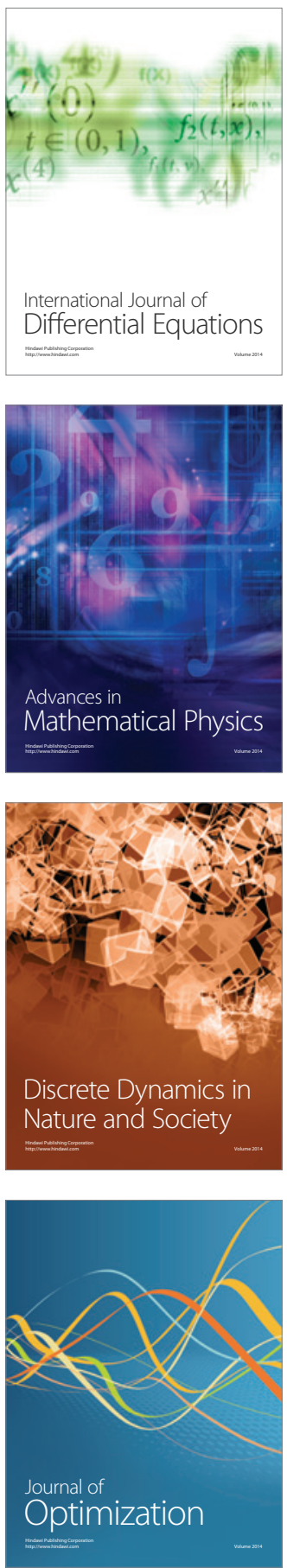